\title{
Self Diffusion and Viscoelasticity in Entangled Systems II. Steady-State Viscosity and Compliance of Binary Blends
}

\author{
Hiroshi FUJITA and Yoshiyuki EINAGA \\ Department of Macromolecular Science, Osaka University, \\ Toyonaka, Osaka 560, Japan
}

(Received June 3, 1985)

\begin{abstract}
A semi-empirical theory for steady-state viscosity $\eta_{0}$ and compliance $J_{\mathrm{e}}$ of a binary blend of homologous monodisperse polymers is formulated by using the expressions for selfdiffusion coefficients derived in Part I and postulating some assumptions for the factors related to the coupling of different polymer chains. It is shown that the expression derived for $\eta_{0}$ can brought to a close numerical agreement with the empirical viscosity law $\eta_{0} \propto M_{w}{ }^{3.4}$, where $M_{w}$ is the weightaverage molecular weight of the polymers making up the blend, if a single function of $x$, the ratio of the molecular weights of the constituent polymers, is suitably chosen. It is also shown that the derived expression for $J_{\mathrm{e}}$ predicts fairly correctly some typical experimental data on polystyrene blends.

KEY WORDS Polymer Blend / Viscosity / Compliance / Self Diffusion Coefficient / Relaxation Spectrum / Plateau Modulus / Chain Coupling /
\end{abstract}

With limited exceptions, the steady-state viscosity $\eta_{0}$ (at zero shear rate) of an amorphous linear polymer in melt or concentrated solution varies linearly with the 3.4 power (or a power close to it) of the polymer's weightaverage molecular weight $M_{w}$, provided that $M_{w}$ is above a certain value $M_{\mathrm{c}}$ characteristic of the system. ${ }^{1}$ The molecular theoretical explanation of this simple empirical viscosity law has attracted many rheologists, but the problem still remains unsolved. Graessley's reviews $^{2,3}$ are instructive for appreciating a variety of approaches to its resolution.

In Part $\mathrm{I}^{4}$ of this series, polymer selfdiffusion in highly entangled systems was formulated by postulating that the dominant motion of chains in such systems is not reputation as proposed by de Gennes ${ }^{5}$ but essentially free and isotropic wriggling and that its rate is substantially controlled by mechanical interactions (not by topological constraints) of different chains through entanglement (the noodle effect). In this paper, the results from Part I are combined with some basic relations in linear viscoelasticity to formulate a semiempirical theory for $\eta_{\mathrm{o}}$ and $J_{\mathrm{e}}$ (the steady-state compliance) of a blend consisting of two homologous monodisperse polymers - the simplest of polydisperse polymer systems. The main aim is to approach something close to, if not entirely identical with, the empirical viscosity law mentioned above.

\section{STEADY-STATE VISCOSITY}

\section{Basic Relations and Assumptions}

Consider a blend consisting of homologous monodisperse polymers 1 and 2 whose chain lengths (measured in the number of segments) $N_{1}$ and $N_{2}$ are so large that the condition $N_{\mathrm{e}}^{\circ} \ll N_{1}, N_{2}$ is satisfied. Here, $N_{\mathrm{e}}^{\circ}$ is the average chain length between entangling points. In what follows, we assume $N_{2}<N_{1}$, and describe the composition of the blend in terms 
of the weight fraction $w$ of polymer 1 .

As usual, any chain in the blend is assumed to deform affinely. Then, the relaxation spectrum $H(\tau)$ of the blend is represented by

$$
H(\tau)=H_{1}(\tau)+H_{2}(\tau)
$$

where $H_{\mathrm{i}}(\tau)(i=1,2)$ is the relaxation spectrum associated with the dynamic response of polymer $i$ in the blend.

According to the theory of linear viscoelasticity, ${ }^{1} \eta_{\mathrm{o}}$ of the blend is given by

$$
\eta_{0}=\int_{0}^{\infty} H(\tau) \mathrm{d} \tau
$$

Substitution of eq 1 gives

$$
\eta_{\mathrm{O}}=A_{1} \mathrm{G}_{\mathrm{N} 1}^{\circ} \tau_{\mathrm{m} 1}+A_{2} \mathrm{G}_{\mathrm{N} 2}^{\circ} \tau_{\mathrm{m} 2}
$$

with

$$
\begin{aligned}
& A_{i}=\left(\int_{0}^{\infty} H_{i} \mathrm{~d} \tau\right)^{2} / \\
& {\left[\left(\int_{0}^{\infty} H_{i} \tau \mathrm{d} \tau\right)\left(\int_{0}^{\infty} H_{i} \tau^{-1} \mathrm{~d} \tau\right)\right] } \\
& G_{\mathrm{N} i}^{\circ}=\int_{0}^{\infty} H_{i} \tau^{-1} \mathrm{~d} \tau \\
& \tau_{\mathrm{m} i}=\int_{0}^{\infty} H_{i} \tau \mathrm{d} \tau / \int_{0}^{\infty} H_{i} \mathrm{~d} \tau
\end{aligned}
$$

The quantity $\tau_{\mathrm{m} i}$ is an average relaxation time for polymer $i$ in the blend and gives a measure of the width of $H_{i}$ over the $\tau$ axis. It seems reasonable to assume (suggested by eq VIII. 14 in ref 5) that

$$
\begin{gathered}
\tau_{\mathrm{m} 1}=B N_{1} / D_{\mathrm{s} 1} \\
\tau_{\mathrm{m} 2}=B N_{2} / D_{\mathrm{s} 2}
\end{gathered}
$$

where $D_{\mathrm{s} i}$ is the (translational) self-diffusion coefficient of polymer $i$ in the blend and $B$ a parameter independent of $N_{1}, N_{2}$, and $w$. Substituting eq 16 and 18 of Part I ${ }^{4}$ for $D_{\mathrm{s} 1}$ and $D_{\mathrm{s} 2}$, respectively, we obtain

$$
\begin{aligned}
\tau_{\mathrm{m} 1}= & B f \zeta_{\mathrm{o}}(k T)^{-1}\left(N_{\mathrm{e}}^{\circ}\right)^{-1.5} \\
& \times\left[w N_{1}+(1-w) N_{2}\right]^{1.5} N_{1}^{2}
\end{aligned}
$$

$$
\tau_{\mathrm{m} 2}=B f \zeta_{\mathrm{o}}(k T)^{-1}\left(N_{\mathrm{e}}^{\circ}\right)^{-1.5} N_{2}^{2.5}
$$

where $\zeta_{0}$ is the segment friction coefficient, $k$ the Boltzmann constant, $T$ the absolute temperature, and $f$ a factor assumed to be independent of $w, N_{1}$, and $N_{2}$.

Instead of $G_{\mathrm{Ni}}{ }^{\circ}$ we introduce $N_{\mathrm{e}(\mathrm{i})}^{\circ}$ defined by

$$
\begin{aligned}
& {G_{\mathrm{N} 1}^{\circ}}^{\circ}=w \rho k T\left(m N_{\mathrm{e}(1)}^{\circ}\right)^{-1} \\
& {G_{\mathrm{N} 2}}^{\circ}=(1-w) \rho k T\left(m N_{\mathrm{e}(2)}^{\circ}\right)^{-1}
\end{aligned}
$$

where $\rho$ is the density of the blend and $m$ the molar mass of a repeating unit of either polymer 1 or 2 .

Substituting eq $9,10,11$, and 12 into eq 3 , we obtain

$$
\begin{aligned}
\eta_{\mathrm{O}}= & C\left\{E_{1} w\left[w N_{1}+(1-w) N_{2}\right]^{1.5} N_{1}{ }^{2}\right. \\
& \left.+E_{2}(1-w) N_{2}^{3.5}\right\}
\end{aligned}
$$

where

$$
C=A^{\bullet} B f \zeta_{\mathrm{o}} m^{-1}\left(N_{\mathrm{e}}^{\circ}\right)^{-2 \cdot 5}
$$

and

$$
E_{\mathrm{i}}=\left(A_{\mathrm{i}} / A^{\bullet}\right)\left(N_{\mathrm{e}}^{\circ} / N_{\mathrm{e}(\mathrm{i})}^{\circ}\right)
$$

with $\mathrm{A}^{\bullet}$ left indeterminate for the time being.

If polymers 1 and 2 in the blend can relax independently, $H_{i}$ is given by $w_{i} H_{i}^{\bullet}$, where $w_{i}$ is the weight fraction of polymer $i$ and $\mathrm{H}_{i}{ }^{*}$ the relaxation spectrum of the same polymer in the pure state. For simplicity, the blend consisting of such polymers is referred to as noninteracting.

The parameter $A_{i}$ for the noninteracting blend is designated by $A_{i}{ }^{\bullet}$. Then we obtain from eq 4

$$
\begin{aligned}
A_{i}^{\bullet}= & \left(\int_{0}^{\infty} H_{i}^{\bullet} \mathrm{d} \tau\right)^{2} / \\
& \times\left[\left(\int_{0}^{\infty} H_{i}^{\bullet} \tau \mathrm{d} \tau\right)\left(\int_{0}^{\infty} H_{i}^{\bullet} \tau^{-1} \mathrm{~d} \tau\right)\right]
\end{aligned}
$$

It is well confirmed ${ }^{1}$ that the shape of the relaxation spectrum in the terminal zone for a series of homologous monodisperse polymers 
is virtually invariant with the chain length $N$ when $N$ is sufficiently larger than $N_{\mathrm{e}}^{\circ}$, i.e., the system is well entangled. From the form of eq 16 we see that $A_{i}{ }^{\circ}$ is essentially determined by the shape of $H_{i}^{\bullet}$ in the terminal zone. Thus, we may conclude that $A_{i}{ }^{\bullet}$ is independent of $N_{i}$ in a good approximation if $N_{i} \gg N_{i}^{\circ}$. Hence, we have good reason to assume

$$
A_{1}^{\bullet}=A_{2}^{\bullet} \equiv A^{\bullet}
$$

under the condition of the present formulation $\left(N_{\mathrm{e}} \bullet \ll N_{1}, N_{2}\right)$. Equation 17 gives the definition of the parameter $A^{\bullet}$ which has been left indeterminate above. With this definition of $A^{\bullet}$ it follows that $A_{i} / A^{\bullet}$ deviates from unity when polymer $i$ undergoes a rheological interaction with the coexisting other polymer in the blend.

For a noninteracting blend we obtain from eq 5,11 , and 12

$$
\rho k T\left(m N_{\mathrm{e}(i)}{ }^{\bullet}\right)^{-1}=\int_{0}^{\infty} H_{i}^{\bullet} \tau^{-1} \mathrm{~d} \tau
$$

where $N_{\mathrm{e}(\mathrm{i})}{ }^{\circ}$ denotes the value of $N_{\mathrm{e}(\mathrm{i})}{ }^{\bullet}$ in this blend. The quantity on the right-hand side of eq 18 is usually equated to what is called the plateau modulus, and it is well known experimentally that this modulus is essentially independent of $N_{i}$ if $N_{i} \gg N_{\mathrm{e}}^{\circ}$. Hence, $N_{\mathrm{e}(\mathrm{i})} \bullet$ may be taken as independent of $N_{i}$ under the condition of the present formulation $\left(N_{\mathrm{e}}^{\circ} \ll N_{1}, N_{2}\right)$; i.e.,

$$
N_{\mathrm{e}(1)}{ }^{\circ}=N_{\mathrm{e}(2)}{ }^{\circ} \equiv N_{\mathrm{e}}^{\circ}
$$

Thus, we see that $N_{\mathrm{e}}^{\circ} / N_{\mathrm{e}(\mathrm{i})}{ }^{\circ}$ in eq 15 should deviate from unity when polymer $i$ undergoes a rheological interaction with the coexisting other chains in the blend.

Summarizing, we find that, under the condition $N_{\mathrm{e}}^{\circ} \ll N_{1}, N_{2}, C$ given by eq 14 may be treated as independent of $N_{1}, N_{2}$, and $w$ and that $E_{i}-1$ may be regarded as the extent to which the relaxation behavior of polymer $i$ in the blend is affected by the coexisting other polymer. In general, $E_{i}$ should depend on $N_{1}$, $N_{2}$, and $w$ as well as the polymer species of the blend.

In Part $\mathrm{I},{ }^{4}$ we showed that self-diffusion of shorter chains in a binary blend is not affected, in a first approximation, by the length of coexisting longer chains. Inferring from this, we postulate that polymer 2 in our blend relaxes as if it were in a noninteracting blend. This is to assume $E_{2}=1$. Then, eq 13 is simplified to

$$
\begin{aligned}
\eta_{\mathrm{O}}=C\left\{E _ { 1 } w \left[w N_{1}\right.\right. & \left.+(1-w) N_{2}\right]^{1.5} N_{1}^{2} \\
& \left.+(1-w) N_{2}^{3.5}\right\}
\end{aligned}
$$

which may be rewritten

$\eta_{\mathrm{O}}=\eta_{\mathrm{O} 2}\left[1-w+w E_{1}(w x+1-w)^{1.5} x^{2}\right]$

where

$$
\begin{gathered}
\eta_{\mathrm{O} 2}=C N_{2}^{3.5} \\
x=N_{1} / N_{2}
\end{gathered}
$$

Since eq 20 reduces to $\eta_{\mathrm{O}}=\mathrm{CN}_{2}{ }^{3.5}$ for $w=0$, $\eta_{\mathrm{O} 2}$ defined by eq 22 represents $\eta_{\mathrm{O}}$ of pure polymer 2 . It can be seen that $\eta_{\mathrm{O} 2}$ varies in proportion to $\mathrm{N}_{2}{ }^{3.5}$, since $\mathrm{C}$ does not depend on $N_{1}$ and $N_{2}$, as mentioned before. The subscript 2 in eq 21 is dummy for a pure polymer, so we find that $\eta_{0}$ for a series of homologous monodisperse polymers increases linearly with $N^{3.5}(N$ is polymer's chain length): $\eta_{\mathrm{O}} \propto N^{3.5}$. This is in substantial agreement with the law established by use of narrow-distribution polymer samples. ${ }^{1}$

For $w=1$, eq 20 should reduce to the equation for $\eta_{\mathrm{O} 1}$, the steady-state viscosity of pure polymer 1 . From this requirement, we obtain $\eta_{\mathrm{O} 1}=C E_{1}(w=1) N_{1}{ }^{3.5}$. Since $\eta_{\mathrm{O} 1}=C N_{1}{ }^{3.5}$ for the reason mentioned above, we find that

$$
E_{1}(w=1)=1
$$

Obviously, $E_{1}$ must be unity for $x=1$, regardless of the value of $w$, because, in this special case, the blend reduces to a pure system. Thus, we have

$$
E_{1}(x=1)=1
$$

For indefinitely large $x$, eq 20 approaches

$$
\eta_{\mathrm{O}}=E_{1}(x=\infty) w^{2.5} \eta_{\mathrm{O} 1}
$$


where the relation $\eta_{\mathrm{O} 1}=C N_{1}^{3.5}$ has been used. It is well recognized by rheologists that when $N_{1} \gg N_{2}$ (i.e., $x \gg 1$ ) and $w$ is not too small, a binary polymer blend in melt behaves rheologically as if it were a concentrated solution of polymer 1 in a low-molecular-weight solvent. ${ }^{1}$ For such solutions the following empirical equation has been established: ${ }^{1}$

$$
\eta_{\mathrm{O}}=K \zeta_{\mathrm{O}}\left(c_{\mathrm{p}}\right) c_{\mathrm{p}}{ }^{3.4} N_{1}{ }^{3.4}
$$

where $\zeta_{\mathrm{o}}\left(c_{\mathrm{p}}\right)$ is the segment friction coefficient as a function of polymer mass concentration $c_{\mathrm{p}}$ and $K$ a constant independent of $N_{1}$ and $c_{\mathrm{p}}$. It follows from eq 27 that

$$
\eta_{\mathrm{O}}=\left[\zeta_{\mathrm{O}}\left(c_{\mathrm{p}}\right) / \zeta_{\mathrm{O}}\right]\left(c_{\mathrm{p}} / \rho\right)^{3.5} \eta_{\mathrm{O} 1}
$$

where we have taken the liberty of changing the index 3.4 to $c_{\mathrm{p}}$ to 3.5 with little risk; $\zeta_{\mathrm{O}}, \rho$ and $\eta_{\mathrm{O} 1}$ have the same meaning as explained before. Comparing eq 26 and 28, with the relation $\mathrm{w} \rho=c_{\mathrm{p}}$ in mind, we obtain

$$
E_{1}(x=\infty)=\left[\zeta_{\mathrm{o}}(w \rho) / \zeta_{\mathrm{O}}\right] w
$$

For our polymer blend, the "solvent" is polymer 2 which is chemically identical with polymer 1 . Hence, $\zeta_{\mathrm{O}}(w \rho)$ is independent of $w$, so that $\zeta_{0}(w \rho) / \zeta_{0}$ can be equated to unity. Thus, if $w$ is not too small,

$$
E_{1}(x=\infty)=w
$$

No other information on $E_{1}$ than eq 24, 25, and 30 can be deduced from general considerations. Therefore, we take a further step by making one more assumption. This is to assume that $E_{1}$ depends on $w$ and $x$, and its dependence on $w$ is linear [anticipating that $E_{1}$ is a mildly varying function of $w(0<w<1)$ ]. So we write $E_{1}$ as

$$
E_{1}=w+\beta(x)(1-w)
$$

which satisfies eq 24 .

By eq 25 and 30 , the unknown function $\beta(x)$ must satisfy the conditions:

$$
\begin{aligned}
& \beta(1)=1 \\
& \beta(\infty)=0
\end{aligned}
$$

the latter of which is valid for $w$ not too close to zero.

With eq 31 substituted, eq 20 takes the form

$$
\begin{aligned}
\eta_{\mathrm{O}} / \eta_{\mathrm{O} 2}= & 1-w \\
& +w[w+\beta(x)(1-w)](w x+1-w)^{1.5} x^{2}
\end{aligned}
$$

Comparison with the 3.4 Power Viscosity Law For our binary blend the empirical 3.4 power viscosity law $\eta_{\mathrm{O}} \propto M_{w}{ }^{3.4}$ gives

$$
\eta_{\mathrm{O}} / \eta_{\mathrm{O} 2}=(w x+1-w)^{3.4}
$$

This gives $\eta_{\mathrm{O}_{1}} / \eta_{\mathrm{O}_{2}}=x^{3,4}$, while eq 34 predicts $\eta_{\mathrm{O} 1} / \eta_{\mathrm{O} 2}=x^{3.5}$. Hence, we replace the exponent 3.4 by 3.5 , and compare

$$
\eta_{\mathrm{O}} / \eta_{\mathrm{O} 2}=(w x+1-w)^{3.5}
$$

with eq 34. However, for this comparison we must know $\beta(x)$. Here we are content with examining how closely eq 34 can be brought to eq 35 by a suitable choice of this function. For this purpose, we choose $\beta(x)$ so that the slopes of $\eta_{\mathrm{O}} / \eta_{\mathrm{O} 2} v s . w$ predicted by eq 34 and 35 agree at $w=1$. Then, we obtain

$$
\beta(x)=2 x^{-1}-x^{-3.5}
$$

Note that eq 36 satisfies eq 32 and 33 . However, this is expected since eq 34 agrees with eq 35 at $x=1$ and $\infty$ when these conditions are fulfilled.

Figure 1 illustrates a comparison of eq 34 and 35 for the case where eq 36 is used in eq 34 . Here, $R$ is defined by

$R=$ Right-hand side (RHS) of eq $34 /$ RHS of eq 35

It can be seen that, for any chosen value of $x$, $R$ does not deviate more than $10 \%$ from unity for $w>0.2$ and that $R$ remains closer to unity over a wider range of $w$ for a larger value of $x$. Probably, usual viscosity measurements on polymer melts with high molecular weight are not more accurate than $\pm 5 \%$. Hence, the result of Figure 1 implies that unless $w<0.2$, 


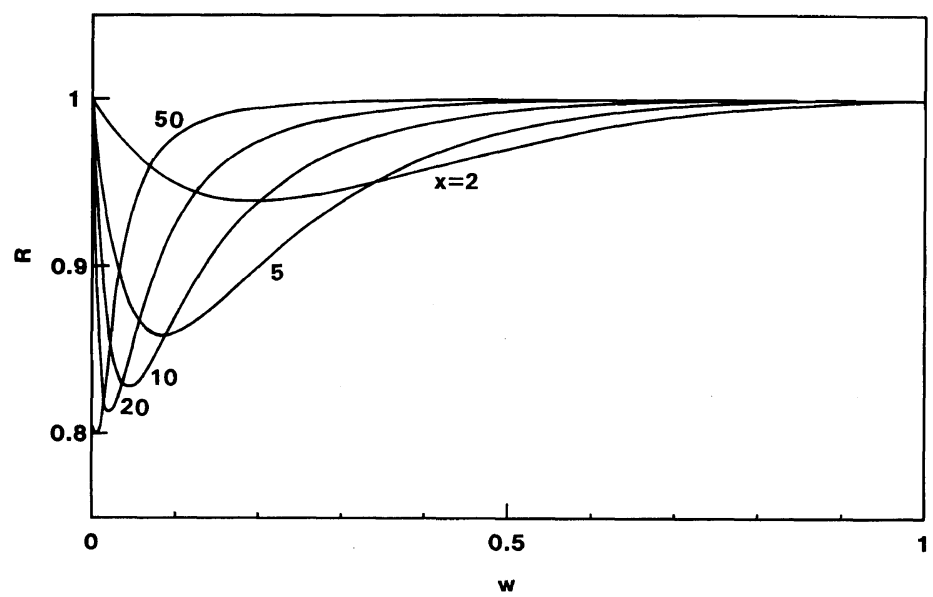

Figure 1. Ratio $R$ plotted against the weight fraction $w$ of polymer 1 for indicated chain length ratios $x$.

eq 34 with $\beta(x)$ given by eq 36 is practically indistinguishable from eq 35 in regard to fitting $\eta_{\mathrm{o}}$ data plotted as a function of $M_{w}$. Of course, we cannot say with this that the reason for the experimental finding of the 3.4 power viscosity law has been elucidated theoretically, since we used this law in advance for the determination of $\beta(x)$. Nonetheless, it seems of theoretical interest that eq 34 can be brought to a close numerical agreement with eq 35 by means of the maneuver taken above.

The marked deviation of $R$ from unity in the region of small $w$ is not unexpected, since, as noted above, eq 33 becomes dubious for such $w$. However, it remains to be seen if they exhibit the shortcoming of the theory developed. As far as we know, little work has yet been done to confirm whether the 3.4 power law is accurately valid or not down to sufficiently small values of $w$, i.e., $M_{w}$ values close to the molecular weight of the shorter chain in a binary blend of homologous polymers.

\section{STEADY-STATE COMPLIANCE}

\section{Basic Relations and Assumptions}

According to the theory of linear viscoelasticity, ${ }^{1} J_{\mathrm{e}}$ for our binary blend can be expressed by

$$
J_{\mathrm{e}}=\eta_{\mathrm{o}}{ }^{-2}\left(A_{1} G_{\mathrm{N} 1}{ }^{\circ} \tau_{\mathrm{m} 1}{ }^{2}+A_{2} G_{\mathrm{N} 2}{ }^{\circ} \tau_{\mathrm{m} 2}{ }^{2}\right)
$$

If appropriate expressions derived and the assumptions made in preceding paragraphs are used, this equation yields

$$
J_{\mathrm{e}}=J_{\mathrm{e} 2} P / Q^{2}
$$

where $J_{\mathrm{e} 2}$ is the steady-state compliance of polymer 2 in the pure state, and $P$ and $Q$ are defined by

$$
\begin{aligned}
& P=1-w+F_{1} w[w+\beta(x)(1-w)](w x+1-w)^{3} x^{4} \\
& Q=1-w+w[w+\beta(x)(1-w)](w x+1-w)^{1.5} x^{2}
\end{aligned}
$$

with

$$
F_{1}=A_{1} / A^{\bullet}
$$

For $w=1$, eq 39 reduces to

$$
J_{\mathrm{e} 1}=J_{\mathrm{e} 2} F_{1}(w=1)
$$

where $J_{\mathrm{e} 1}$ is the steady-state compliance of polymer 1 in the pure state. Since, by eq 16 , $F_{1}(w=1)=1$, we find

$$
J_{\mathrm{e} 1}=J_{\mathrm{e} 2}
$$

Thus, under the condition of the present formulation $\left(N_{\mathrm{e}}^{\circ} \ll N_{1}, N_{2}\right), J_{\mathrm{e}}$ of a pure polymer is independent of chain length.

For $x=1, \beta$ is equal to unity (see eq 32 ), so 
that eq 39 gives

$$
J_{\mathrm{e}}(x=1)=J_{\mathrm{e} 2}\left[1-w-F_{1}(x=1) w\right]
$$

Since $J_{\mathrm{e}}(x=1)$ is $J_{\mathrm{e} 2}$ for any $w$, we find that

$$
F_{1}(x=1)=1
$$

regardless of the value of $w$.

Finally, since $\beta(\infty)=0$ (see eq 33 ), eq 39 reduces to

$$
J_{\mathrm{e}}(x=\infty)=J_{\mathrm{e} 1} F_{1}(x=\infty) w^{-2}
$$

where the relation $J_{\mathrm{e} 1}=J_{\mathrm{e} 2}$ has been used.

As mentioned before, when $x \gg 1$, the rheological behavior of our polymer blend is similar to that of a concentrated solution of polymer 1 in a low-molecular-weight solvent. Experimentally, $J_{\mathrm{e}}$ of such a solution is known to vary with polymer mass concentration $c_{\mathrm{p}}$ as ${ }^{1}$

$$
J_{\mathrm{e}} \propto c_{\mathrm{p}}{ }^{-2}\left(c_{\mathrm{p}} \text { is not too low }\right)
$$

with the proportionality factor independent of chain length. From eq 47 we obtain

$$
J_{\mathrm{e}}=\left(\rho / c_{\mathrm{p}}\right)^{2} J_{\mathrm{e} 1}
$$

where, as defined above, $J_{\mathrm{e} 1}$ is the steady-state compliance of pure polymer 1 and $\rho$ the density of the same polymer. Applying eq 48 to our blend with $x=\infty$, we may equate $J_{\mathrm{e}}$ to $J_{\mathrm{e}}$ $(x=\infty)$ and $\rho / c_{\mathrm{p}}$ to $w^{-1}$. Thus, a comparison of eq 48 with eq 46 gives

$$
F_{1}(x=\infty)=1
$$

which is subjected to the condition that $w$ is not too small.

On the basis of the three properties of $F_{1}$ deduced above, we assume that $F_{1}=1$ for any values of $x$ and $w$, excepting $w \cong 0$. If this assumption is accepted, it turns out that deviations of the parameter $E_{1}$ (see eq 15) from unity arise from the rheological effect of polymer 2 on $N_{\mathrm{e}}^{\circ} / N_{\mathrm{e}(1)}^{\circ}$.

In Figure 2, differently marked points indicate published data of $J_{\mathrm{e}} / J_{\mathrm{e} 1}$ for some binary blends of narrow-distribution polystyrene, and the solid curves show this ratio computed from eq 39 with $F_{1}=1, J_{\mathrm{e} 1}=J_{\mathrm{e} 2}$, and $\beta(x)$ given by eq

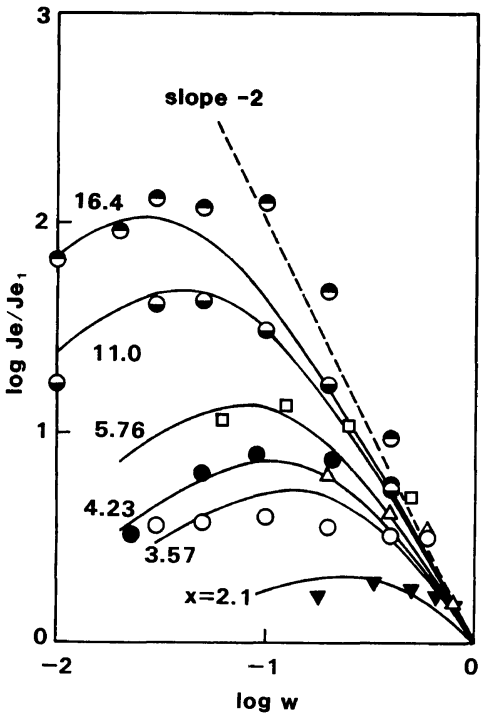

Figure 2. Ratio $J_{\mathrm{e}} / J_{\mathrm{el}}$ of binary polystyrene blends double-logarithmically plotted against the weight fraction $w$ of polymer 1 for indicated chain length ratios $x$. $\ominus, M_{1}=1.19 \times 10^{6}, M_{2}=7.24 \times 10^{4} ; \ominus, M_{1}=4.27 \times 10^{5}$, $M_{2}=3.89 \times 10^{4}\left(\right.$ Watanabe and Kotaka $\left.{ }^{7}\right) ; \square, M_{1}=$ $5.00 \times 10^{5}, M_{2}=8.68 \times 10^{4}\left(\right.$ Mills and Nevin $\left.{ }^{8}\right) ; \bigcirc, M_{1}=$ $4.11 \times 10^{5}, M_{2}=9.72 \times 10^{4}\left(\right.$ Prest $\left.^{9}\right) ; \triangle, M_{1}=1.67 \times 10^{5}$, $M_{2}=4.69 \times 10^{4}$ (Masuda et al. $\left.{ }^{6}\right) ; \bigcirc, M_{1}=3.16 \times 10^{5}$, $M_{2}=8.86 \times 10^{4}\left(\right.$ Watanabe et $\left._{\text {al }}{ }^{10}\right) ; \nabla, M_{1}=2.54 \times 10^{5}$, $M_{2}=1.21 \times 10^{5} \quad\left(\right.$ Akovali $\left.^{11}\right)$. Solid curves represent theoretical predictions.

36 for the $x$ values which characterize the respective blends. Except for $x=1$, these curves become tangent to the dashed line of slope -2 as $w$ approaches unity. Taking the difficulty of accurate determination of $J_{\mathrm{e}}$ into account, we may consider that the predictability of the present theory concerning the $x$ and $w$ dependence of $J_{\mathrm{e}}$ is fairly good. In particular, the theory predicts rather correctly the maximum of $J_{\mathrm{e}} / J_{\mathrm{e} 1}$ observed for each $x$. However, the theoretical maxima occur systematically at the $w$ values somewhat lower than those estimated from the plotted points. Moreover, the limiting slope of $\log J_{\mathrm{e}} / J_{\mathrm{e} 1} v s . \log w$ at $\log w=0$ may be concluded from these data to be somewhat higher than the theoretical -2 , as has been claimed by Masuda et al. ${ }^{6}$ 


\section{CONCLUDING REMARKS}

It is well recognized that rheological properties of a polymer blend cannot quantitatively be predicted in terms of those of its components in the pure state. This implies that the coupling, both mechanical and topological, of different polymer chains plays a decisive role in determining rheological behavior of condensed polydisperse polymers. As far as we know, attempts to establish a "blending law" by taking this effect phenomenologically into account have achieved no more than limited success. The semi-empirical theory developed above may be considered an example of such an attempt. Its success as seen above, if not full, reflects the fact that the coupling effect has been more properly formulated than before. We say that the use of the expressions for $D_{\text {si }}$ derived in Part I and the approximations made for $E_{i}$ are primarily responsible for this.

\section{REFERENCES}

1. J. D. Ferry, "Viscoelastic Properties of Polymers," 3rd ed, Wiley-Interscience, New York, N. Y., 1980.

2. W. W. Graessley, Adv. Polym. Sci., 16, 1 (1974).

3. W. W. Graessley, Adv. Polym. Sci., 47, 67 (1982).

4. H. Fujita and Y. Einaga, Polym. J., 17, 1131 (1985); Nihon Reoroji Gakkaishi, 12, 136 (1984).

5. P. G. de Gennes, J. Chem. Phys., 55, 572 (1972); "Scaling Concepts in Polymer Physics," Cornell Univ. Press, Ithaca, N. Y., 1979.

6. T. Masuda, K. Kitagawa, T. Inoue, and S. Onogi, Macromolecules, 2, 116 (1970).

7. H. Watanabe and T. Kotaka, Macromolecules, 17, 2316 (1984).

8. N. J. Mills and A. Nevin, J. Polym. Sci., A-2, 9, 267 (1971).

9. W. M. Prest, Jr., Polym. J., 4, 163 (1973).

10. H. Watanabe, T. Sakamoto, and T. Kotaka, Macromolecules, in press.

11. G. Akovali, J. Polym. Sci., A-2, 5, 875 (1967). 